\title{
Treating co-morbid insomnia and social anxiety disorder with sequential CBT protocols: a single-case experimental study
}

\author{
Daniel Wallsten ${ }^{1 \star(D)}$, Annika Norell-Clarke ${ }^{1,2}$ (D), Sven Alfonsson ${ }^{4}$, David Gryphon ${ }^{3}$, \\ Hanna Eriksson ${ }^{3}$ and Maria Tillfors ${ }^{1}$ \\ ${ }^{1}$ Department of Social and Psychological Studies, Karlstad University, Karlstad, Sweden, ${ }^{2}$ Faculty of Health and Science, \\ Kristianstad University, Kristianstad, Sweden, ${ }^{3}$ Department of Law, Psychology and Social Work, Örebro University, \\ SE-70182 Örebro, Sweden and ${ }^{4}$ Centre for Psychiatry Research, Department of Clinical Neuroscience, Karolinska \\ Institutet and Stockholm Health Care Services, 113 64, Stockholm, Sweden \\ *Corresponding author. Email: daniel.wallsten@kau.se
}

(Received 28 June 2020; revised 6 April 2021; accepted 5 May 2021; first published online 09 July 2021)

\begin{abstract}
Background: Although insomnia disorder and social anxiety disorder are among the most prevalent psychiatric disorders, no studies have yet evaluated the use of sequential evidence-based treatment protocols in the population with co-morbid social anxiety disorder and insomnia disorder.

Aims: This study aimed to investigate the effects of sequential treatments on co-morbid insomnia disorder and social anxiety disorder. As depression is a common co-morbid syndrome for both insomnia and social anxiety, a secondary aim was to examine depressive symptoms.

Method: A single-case repeated crossover AB design was used. Ten participants between 18 and 59 years of age with co-morbid DSM-5 diagnoses of insomnia disorder and social anxiety disorder received sequential treatments with cognitive behavioural therapy (CBT). Seven participants completed the treatment course. The primary outcomes were symptoms of insomnia and social anxiety, and the secondary outcome was symptoms of depression.

Results: The effects of CBT on people with co-morbid social anxiety disorder and insomnia disorder were mixed. The majority of participants improved their sleep quality and lessened symptoms of social anxiety and depression. However, participants differed in their degree of improvement concerning all three disorders.

Conclusions: Sequential CBT treatments are potentially effective at decreasing symptoms of social anxiety and insomnia for people with co-morbid social anxiety disorder and insomnia disorder. The variation in outcome across participants makes firm conclusions about the treatment efficacy difficult to draw.
\end{abstract}

Keywords: cognitive behavioural therapy; co-morbidity; depression; insomnia; single case experimental design; social anxiety

\section{Introduction}

Co-morbidity of psychiatric disorders, as defined by the Diagnostic and Statistical Manual of Mental Disorders (DSM, 5th edition; American Psychiatric Association, 2013) are very common (Kessler et al., 2005; Roca et al., 2009). Nevertheless, knowledge on the most effective treatments for certain combinations of disorders is lacking, as reflected by the scarcity of publications. Co-morbid insomnia disorder and social anxiety disorder is one such

(c) The Author(s), 2021. Published by Cambridge University Press on behalf of the British Association for Behavioural and Cognitive Psychotherapies. This is an Open Access article, distributed under the terms of the Creative Commons Attribution licence (http:// creativecommons.org/licenses/by/4.0/), which permits unrestricted re-use, distribution, and reproduction in any medium, provided the original work is properly cited. 
understudied psychiatric combination, with only one identified case study $(n=1)$ investigating treatments for this co-morbidity (Tang, 2010).

Insomnia is characterized by sleep onset latency, undesired periods of wakefulness during the night, or early awakenings combined with impaired functioning during daytime. Approximately $25-30 \%$ of the general population reports at least one criterion for insomnia disorder, while 610.5\% meet the diagnostic criteria (American Psychiatric Association, 2013; Mallon et al., 2014; Ohayon, 2002).

Social anxiety disorder is one of the most common anxiety disorders across western populations, with a lifetime prevalence of approximately 13\% (Bandelow and Michaelis, 2015). Social anxiety disorder is characterized by persistent fear of underperforming in social situations, being negatively judged by others, and impairment in everyday functioning through avoidance (American Psychiatric Association, 2013). Insomnia has historically been considered symptomatic of other psychiatric disorders, as reflected by earlier versions of the DSM. However, more recent research indicates a complex and bi-directional relationship between insomnia and anxiety disorders (Blake et al., 2018; Krystal, 2012).

First, the presence of either insomnia or an anxiety disorder seems to predict an increased incidence of the other disorder (LeBlanc et al., 2009; Morphy et al., 2007; Neckelmann et al., 2007). People suffering from insomnia disorder are twice as likely to develop anxiety disorders relative to healthy controls (Krystal, 2012) and disturbed sleep has been proposed to increase fear responses and negatively affect emotion regulation, thereby worsening symptoms of anxiety (Babson et al., 2009; Walker and van der Helm, 2009). Second, research on the relationship between insomnia disorder and social anxiety disorder indicates that the presence of social anxiety predicts greater insomnia disorder severity (Buckner et al., 2008). However, a review found no significant differences in sleep parameters between people with social phobia and healthy controls (Papadimitriou and Linkowski, 2005). Furthermore, Uhde et al. (2009) described this combination as 'much less frequent' than co-morbidity between insomnia and other anxiety disorders. Finally, Buckner et al. (2008) proposed that the relationship between insomnia disorder and symptoms of social anxiety is mediated by major depressive disorder. To summarize, the relationship between insomnia and social anxiety disorder seems to be somewhat more nuanced than the relationships between insomnia and other anxiety disorders; nevertheless, further research is warranted.

Cognitive behavioural therapy (CBT) has been found to be a relatively effective treatment for both social anxiety and insomnia disorder (Rodebaugh et al., 2004; van Straten et al., 2018). A meta-analysis found that $36 \%$ of patients with insomnia and co-morbid psychiatric or medical disorders receiving CBT for insomnia (CBT-I) reached remission, and the effect sizes for most symptoms and sleep parameters varied between medium and large (Wu et al., 2015). Similarly, van Straten et al. (2018) reported robust and similar results for CBT-I on symptoms of insomnia disorder with and without co-morbidity. However, social anxiety disorder was not included among other co-morbid anxiety disorders, and it remains unknown whether or how symptoms of insomnia are affected by CBT for social anxiety disorder (CBTS; Belleville et al., 2010). One study suggested that treating social anxiety does not affect sleep problems (Kushnir et al., 2014). Similarly, treatment of insomnia disorder seems to affect symptoms of anxiety only moderately (Belleville et al., 2011; Tang, 2010). Poorer sleep quality at baseline, however, predicts a longer recovery period and poorer treatment outcomes of CBTS on social anxiety disorder (Zalta et al., 2013). In a systematic review, Eskildsen et al. (2010) concluded that data on co-morbidity as a predictor for drop-out and outcome when treating social anxiety disorder were somewhat inconclusive for major depressive disorder but not for anxiety disorders, where no such relationship was found. No studies exploring insomnia as a predictor for outcome or treatment of social anxiety disorder were identified.

In conclusion, insomnia disorder and social anxiety disorder are common psychiatric disorders whose relationship requires further clarification. The robust results for CBT-I and CBT-S and the 
unclear relationship between the disorders suggests that the use of sequential protocols in accordance with the current evidence base may be a feasible strategy to treat co-morbidity of insomnia disorder and social anxiety disorder. No studies have yet investigated the use of both CBT-I and CBT-S on patients with this combination of disorders. This study was designed to explore the effects of sequential CBT treatments on these disorders through the following questions: (1) does sequential treatment based on CBT-S and CBT-I improve symptoms of social anxiety disorder for people with co-morbid insomnia disorder?; (2) does sequential treatment based on CBT-S and CBT-I improve symptoms of insomnia disorder for people with co-morbid insomnia disorder?; and (3) does sequential treatment based on CBT-S and CBT-I decrease depressive symptoms?

\section{Method}

\section{Design}

A single-case repeated crossover $A B$ design was used in which the baseline measurement phase (A) preceded the treatment phase (B). The repeated measurements during phase A allows for predictions to be made about current symptoms in the absence of treatment, and can be compared with the measurements during treatment in phase $\mathrm{B}$. The combination of repeated measurements in both phases, randomization to the different sequences, and the replication across participants allow for exploration of order effects, testing effects, and regression towards the mean (Kazdin, 2011). Participants were randomized into two different treatment sequences for phase B: CBT-S followed by CBT-I (sequence 1) and CBT-I followed by CBT-S (sequence 2). Participants completed daily and weekly self-rating scales during phase A, phase $\mathrm{B}$, at post-treatment (sequence 1, one week after treatment, and sequence 2 four weeks after treatment due to practical constraints), and at the 4-month follow-up.

\section{Participants and procedure}

Participants were recruited through advertisements placed in local media, social media, through email lists to university students, and through counsellors at primary healthcare centres. The included participants ( $n=10$; Fig. 1) met the DSM-5 criteria for social anxiety disorder and insomnia disorder but not for other primary sleep disorders such as sleep apnea (American Psychiatric Association, 2013). All participants were $\geq 18$ years of age, not at high risk of suicide [as indicated by $\geq 4$ on the Montgomery-Åsberg Depression Rating Scale-Self-Rated (MADRS-S)] and did not suffer from more severe psychiatric disorders like severe depression (total score of $\geq 30$ on MADRS-S) or manic states [indicated by Mini International Neuropsychiatric Interview (MINI)]. The participants did not receive any other concurrent psychological treatment and had not changed their medication regimen within the past three months (Table 1).

\section{Measures}

All questionnaires and structured interviews were found to have satisfactory psychometric properties (Amorim et al., 1998; Baker et al., 2002; Bastien et al., 2001; Carney et al., 2009; Fantino and Moore, 2009; Fresco et al., 2001; Furmark et al., 1999; Lecrubier et al., 1997; Morin et al., 2011; Sheehan et al., 1997; Sheehan et al., 1998; Svanborg and Asberg, 1994).

The MINI (Sheehan et al., 1998) with modified criteria for social anxiety disorder according to DSM-5 was used to assess psychiatric disorders during screening (American Psychiatric 


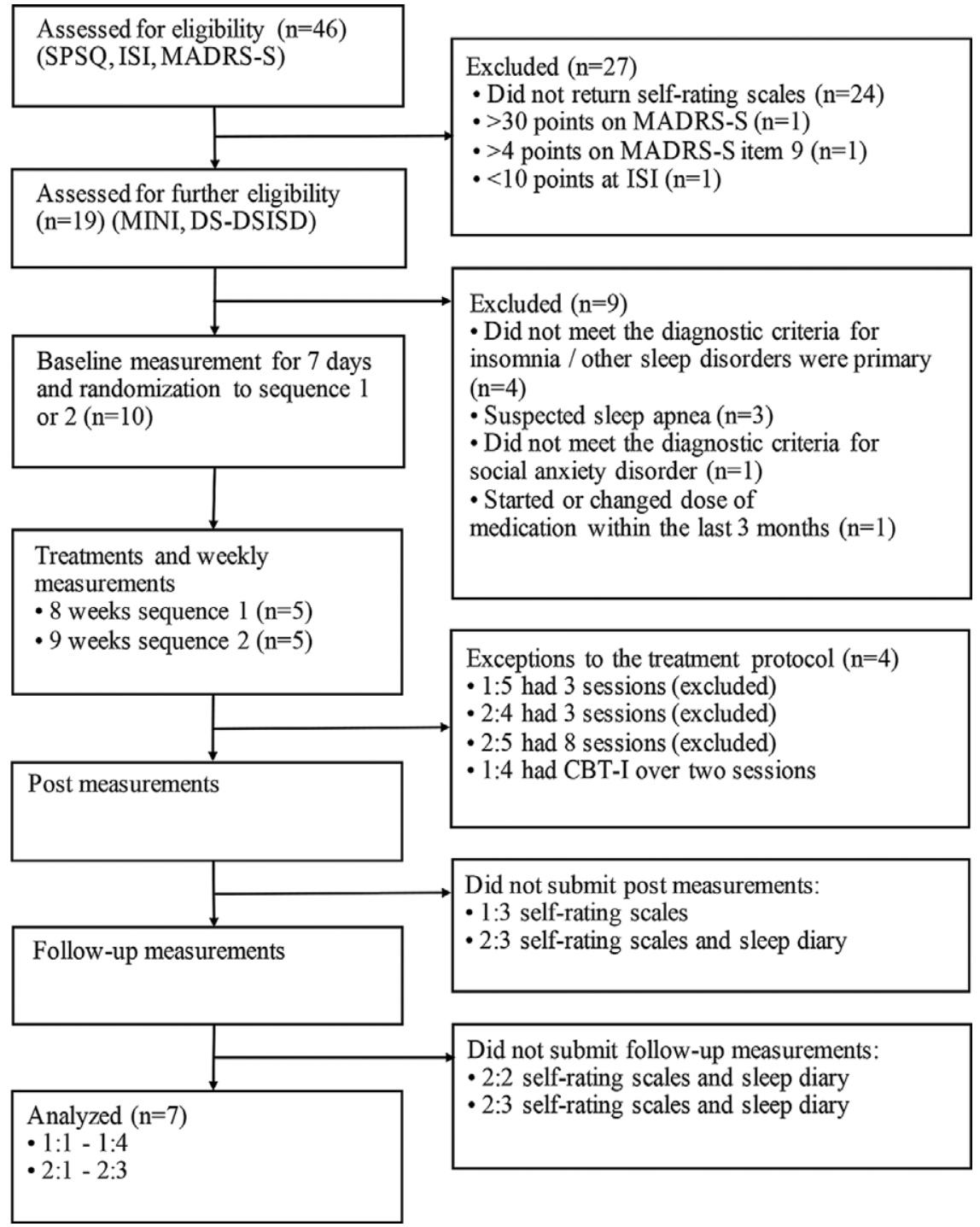

Figure 1. Flowchart of participants.

Association, 2013). The Duke Structured Interview for Sleeping Disorders (DSISD; Carney et al., 2009) was used to assess clinical levels of current sleep disorders according to the DSM-IV and the ICSD-2, with updated DSM-5 criteria for insomnia disorder (American Psychiatric Association, 2013). Both interviews were conducted over the telephone.

\section{Self-rating scales}

The Social Phobia Screening Questionnaire (SPSQ; Furmark et al., 1999) was administered during the screening procedure and was used alongside the MINI to assess social anxiety disorder. The scale includes 14 items consisting of situations related to social interaction or social performance that are graded from 0 (not at all distressing) to 4 (extremely distressing). The total score ranges from 0 to 56. The assessment of clinical levels was performed according to the instrument's 
Table 1. Demographics

\begin{tabular}{|c|c|c|c|c|c|c|c|c|}
\hline Participant & Sex & Age range & Relationship status & Highest education & Employ-ment & Pharmacological treatment & $\begin{array}{l}\text { Other psychiatric conditions } \\
\text { diagnosed with MINI }\end{array}$ & Completed treatment \\
\hline $1: 1$ & Female & $18-29$ & Single & High school & Yes & No & $\begin{array}{l}\text { Recurrent major depressive } \\
\text { disorder, agoraphobia }\end{array}$ & Yes \\
\hline $1: 2$ & Male & $50-59$ & Partner & High school & Yes & No & - & Yes \\
\hline $1: 3$ & Female & $40-49$ & Single & High school & Yes & No & - & Yes \\
\hline $1: 4$ & Female & $18-29$ & Partner & High school & Yes & No & Generalized anxiety disorder & Yes \\
\hline 1:5 & Female & $18-29$ & Partner & High school & Yes & No & $\begin{array}{l}\text { Agoraphobia, generalized } \\
\text { anxiety disorder }\end{array}$ & No \\
\hline $2: 1$ & Female & $18-29$ & Partner & High school & Yes & No & $\begin{array}{l}\text { Generalized anxiety disorder, } \\
\text { primary agoraphobia }\end{array}$ & Yes \\
\hline $2: 2$ & Female & $18-29$ & Partner & University & Yes & No & Major depressive disorder & Yes \\
\hline $2: 3$ & Female & $18-29$ & Single & High school & Yes & No & $\begin{array}{l}\text { Major depressive disorder, } \\
\text { primary agoraphobia }\end{array}$ & Yes \\
\hline $2: 4$ & Male & $30-39$ & Single & High school & Yes & No & $\begin{array}{l}\text { Major depressive disorder, } \\
\text { generalized anxiety } \\
\text { disorder }\end{array}$ & No \\
\hline 2:5 & Male & $30-39$ & Partner & University & Yes & No & - & No \\
\hline
\end{tabular}

Age ranges were used to maintain participant anonymity. 
guidelines, namely, 0-16: probably no social phobia, 17-21: risk zone, $>21$ : probably social phobia, >29: probably generalized social phobia.

The Liebowitz Social Anxiety Scale-Self Report (LSAS-SR; Baker et al., 2002) was used to assess symptoms of social anxiety. This report consists of 24 different social interaction and performance situations that are graded according to the level of induced anxiety and avoidance, respectively, from 0 (no fear/anxiety and no avoidance) to 3 (severe fear/anxiety and severe avoidance). The total score ranges from 0 to 144 . Assessment of patient estimates followed the instrument's guidelines, where scores $>30$ indicate probable social phobia, while scores $>60$ indicate probable generalized social phobia. The LSAS-SR was administered three times over 7 days during baseline (A), weekly during treatment (B), once post-treatment, and once at the 4-month follow-up.

A modified short version of the Social Phobia Rating Scale (SPRS; Wells and Leahy, 1998) was used to collect daily data on social anxiety. The first three of the five original items were used, which assessed the past 24 hours instead of the past week. The three items consisted of questions regarding how distressing or disabling social anxiety has been, how much social situations have been avoided due to anxiety, and degree of self-consciousness in difficult social situations. The items are rated on an 8-point scale, where low numbers indicate lower extents of distress, avoidance and self-consciousness. The modified version of the SPRS was administered daily during baseline (A) and treatment (B), once daily for a 1-week period post-treatment and once daily for a 1 -week period at the 4-month follow-up. A short scale with a relatively wide range of answer options was considered feasible for daily measurements. The modified version of the SPRS was used as the primary measure of social anxiety disorder.

The Insomnia Severity Index (ISI; Bastien et al., 2001) was used to assess symptoms of insomnia disorder. Its seven items are each answered on a 5-point scale, where higher points indicate more severe difficulties. The total score ranges from 0 to 28 (Bastien et al., 2001; Morin et al., 2011). Assessment of participant estimates followed the guidelines of Morin et al. (2011), which suggested that a total score $\geq 10$ is indicative of clinical insomnia. ISI was administered three times over 7 days during baseline (A), once weekly throughout the treatment period (B), once at post-treatment, and once at the 4-month follow-up.

The Montgomery-Åsberg Depression Rating Scale-Self-Rated (MADRS-S; Svanborg and Asberg, 1994) was used to assess depressive symptoms. Nine items are answered on a 7-point scale, with a total possible score of 54 . The clinical categories used were: 0-12: normal/ symptoms absent, 13-19: mild depression, 20-34: moderate depression, and >34: severe depression ( $\geq 30$ was mistakenly used in line with the clinician-administered version to indicate severe depression during screening). The MADRS-S was administered three times over 7 days during baseline (A), once weekly throughout the treatment period (B), once at post-treatment, and once at the 4-month follow-up.

A sleep diary was used to assess sleep patterns. Data consisted of time points for bedtime, last awakening, and time out of bed, but also sleep onset latency (SOL; minutes between going to bed and first sleep onset), wake after sleep onset (WASO; minutes awake after first sleep onset), early morning awakening (EMW; minutes in bed after last awakening), and number of awakenings. Undesired time awake (SOL+WASO) was calculated and used as a primary measure. The sleep diary was administered daily during baseline (A) and treatment (B), once daily for a 1 -week period post-treatment and once daily for a 1-week period at the 4month follow-up.

\section{Treatment}

Each participant received 10 treatment sessions: four CBT-I sessions and six CBT-S sessions. Sequence 1 (CBT-S before CBT-I) ran over 9 weeks, and sequence 2 (CBT-I before CBT-S) ran over 8 weeks. The discrepancy in treatment duration was a result of practical constraints. 
The CBT-I was a modified version of the Edinger and Carney (2014) protocol. The Swedish translation was made by A. Norell-Clarke (unpublished) and has been used in other CBT-I studies (Norell-Clarke et al., 2015). The protocol consists of two blocks over four sessions. The first block touched on behavioural aspects, such as sleep restriction and stimulus control, while the second block addressed cognitive aspects, such as constructive worry, thought registration and cognitive distortions. As CBT-S included tracking and self-registration of negative automatic thoughts, it was removed from the second block of CBT-I to avoid overlap. Sleep efficiency (effective sleep time divided by total time in bed) was calculated from the participant's sleep diary. Sleep efficiency between 80 and $85 \%$ was used as a threshold during sleep restriction (Edinger and Carney, 2014).

The CBT-S was a protocol based on a modified version of a treatment administered online (Furmark et al., 1999; Tillfors et al., 2008). The original protocol was modified to accommodate six individual sessions and included psychoeducation, cognitive restructuring, including tracking and self-registration of negative automatic thoughts (sessions 1-3), behavioural experiments with and without safety behaviours (session 3), exposure (sessions 4-6), and relapse prevention (session 6). The participants completed related assignments at home between sessions in both treatment groups.

The therapists were two Master's students in clinical psychology in their final year of education (D.G. and H.E.). They received supervision from a clinical psychologist and professor in clinical psychology (M.T., an expert on social anxiety disorder) and a clinical psychologist with a $\mathrm{PhD}$ in psychology (A.N.-C., an expert on insomnia).

\section{Data analysis}

\section{Daily measures}

Social anxiety and undesired time awake were analysed primarily visually (see Figs 2 and 3), which is considered the standard procedure for analysing data in a single case design (Kazdin, 2011). Median values, rather than means, were calculated for the baseline phase (phase A), treatment phase (phase B), post-treatment phase, and follow-up phase in order to handle fluctuations with extreme outliers.

Non-overlap of all pairs (NAP) was used to calculate phase differences and effect sizes for the daily measures of social anxiety (modified version of SPRS) and undesired time awake (SOL+ WASO; Manolov et al., 2016; Parker and Vannest, 2009; Parker et al., 2011). All data points in phase A were individually compared with all individual data points in phase B; thus, NAP shows the percentage of data that improve across phases. The NAP values were transformed to adjust for chance level ( 1 - NAP/0.5), with negative values indicating deterioration. The proportion of non-overlaps can be interpreted as follows: weak effects: 0 -..31; medium effects: .32-.84; large or strong effects: .85-1.0 (Parker and Vannest, 2009).

For weekly measures, the mean scores for each phase and scale, as well as the differences between baseline means and post-treatment scores, were calculated. The baseline phase (A) consisted of three measures over 7 days, and the treatment phase (B), comprising CBT-I and CBT-S, consisted of weekly measures. The scales used for weekly measures were administered once during post-treatment data collection and once during the 4-month follow-up. Data from missing single items (10 data points across all subjects) were replaced with the mean for the previous and the following weekly measure of that same item, in order to not lose all data from that measurement. More advanced imputation methods were considered superfluous given the few missing data points for single items. Imputation was not conducted when data from more than single items were missing. The data from participants 1:1-4 and 2:1-3 were analysed. Due to missing data and low adherence, data from participants 1:5, 2:4, and 2:5 were excluded from the analysis (Fig. 1). 

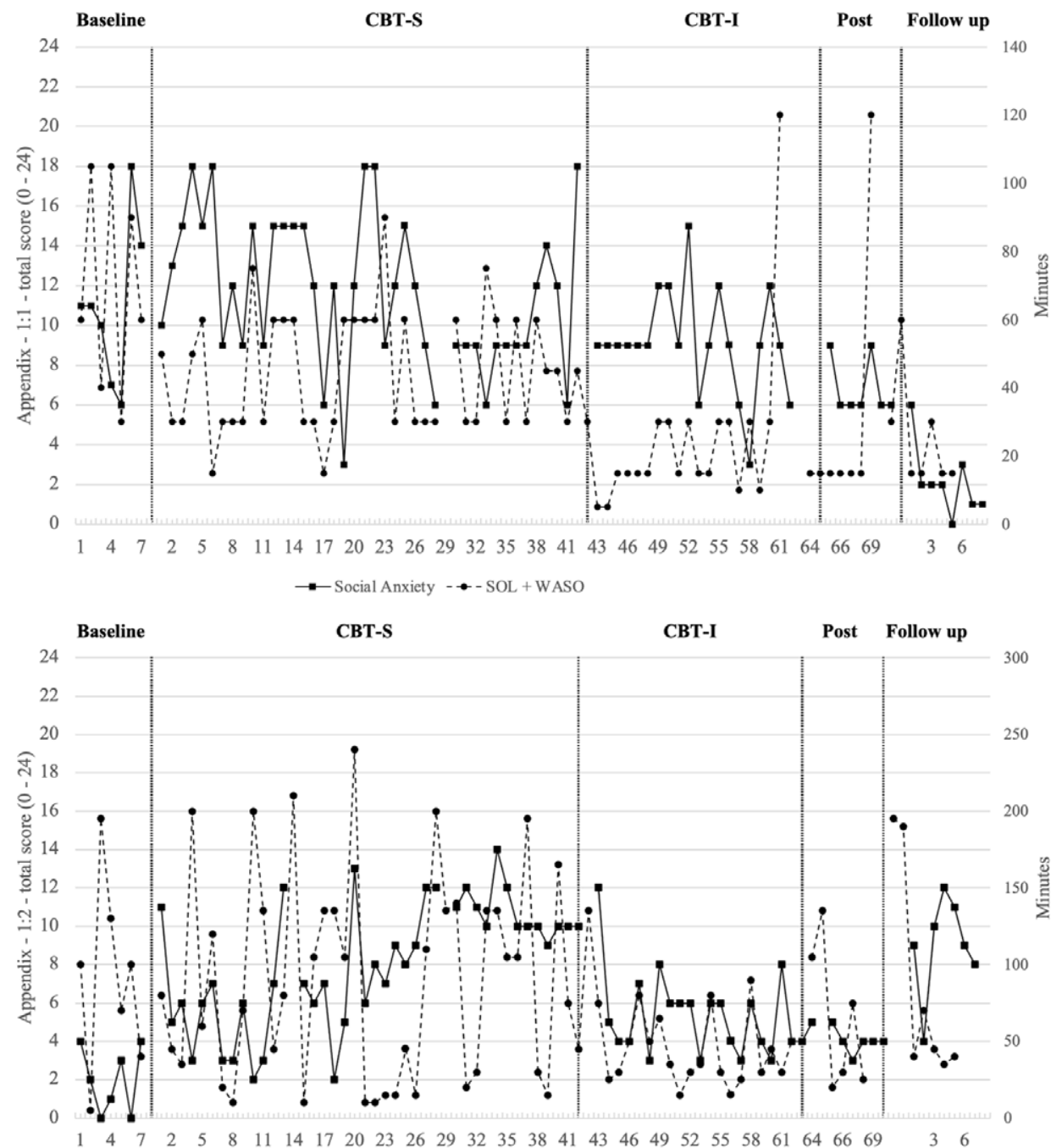

$\rightarrow-$ Social Anxiety $\quad-\bullet$ SOL + WASO

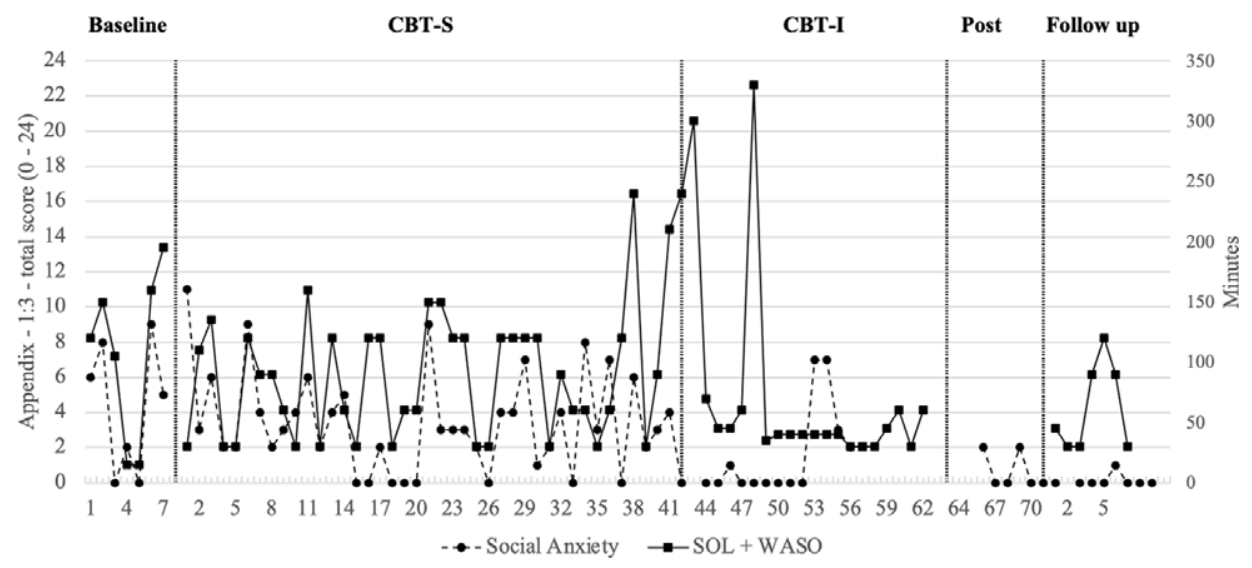

Figure 2. Social anxiety and insomnia symptoms over time for treatment sequence 1 . 


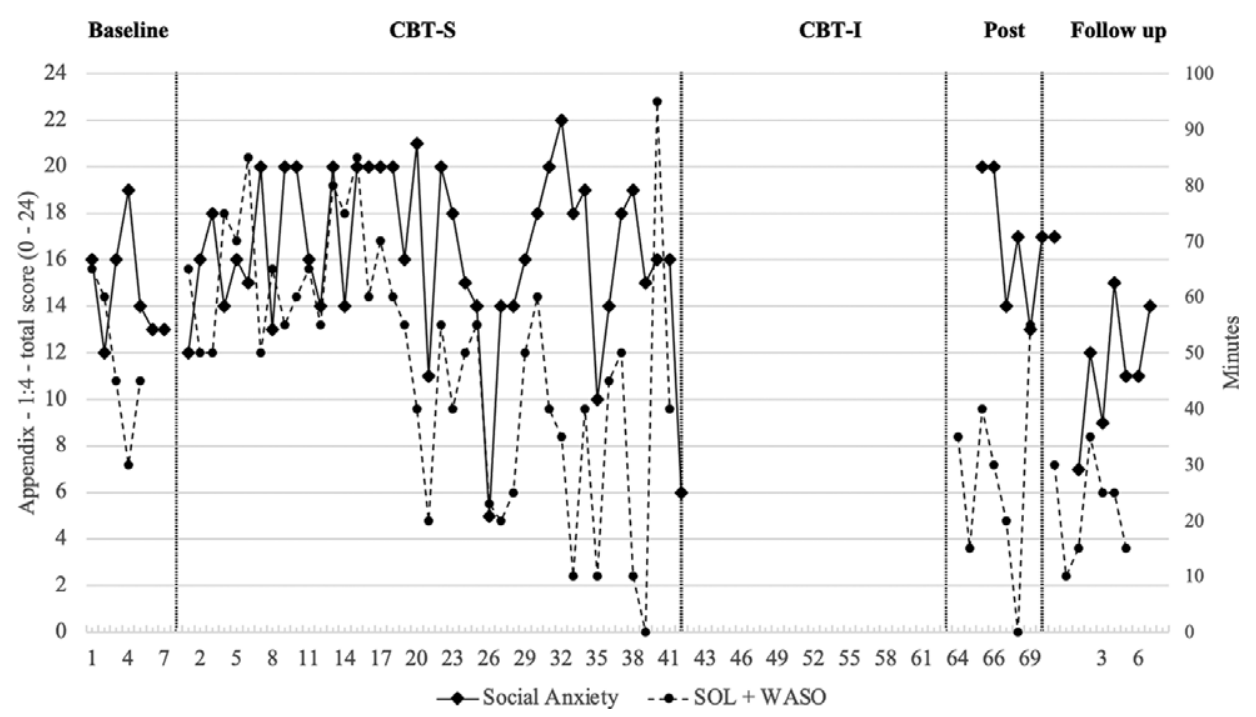

Figure 2. (Continued).

\section{Results}

Does sequential treatment based on CBT-S and CBT-I improve symptoms of social anxiety disorder for people with co-morbid insomnia disorder?

Visual analyses of data from the daily social anxiety measures (with a modified version of SPRS) from participants 1:1, 1:3, 2:1 and 2:2 indicated a decrease in reported symptoms between the baseline phase (A) and treatment phase (B). The reported symptoms were either stable or had improved from post-treatment to the 4-month follow-up. Follow-up data for participant 2:2 were not obtained (see Figs 2 and 3). Calculations with NAP corroborated the medium-tolarge effect sizes seen in the visual analysis (see Table 2). Further, the weekly social anxiety measures (LSAS-SR) showed symptom reductions for participants 1:1, 1:3, 2:1 and 2:2.; however, for participants $2: 1$ and 2:2, the symptom reductions did not reach non-clinical levels (i.e. $<30$ ), which was the case for participants $1: 1$ and 1:3 at follow-up (see Table 3).

The daily social anxiety data from participants 1:2, 1:4 and 2:3 indicated an increase in reported symptoms between the baseline (A) and treatment (B) phases. However, the reported symptoms for participants 1:4 improved from post-treatment to the 4-month follow-up (see Figs 2 and 3). Neither the post-treatment data nor the follow-up data were obtained for participant 2:3. The NAP scores for these three participants corroborated the visual analysis (see Table 2). The weekly social anxiety measures (LSAS-SR) for participant 2:3 showed similar patterns with increased reported symptoms. For participants 1:2 and 1:4, however, the weekly social anxiety measures instead indicated slight improvements (see Table 3).

\section{Does sequential treatment based on CBT-S and CBT-I improve symptoms of insomnia disorder for people with co-morbid social anxiety disorder?}

Visual analyses of data from the daily insomnia measures (SOL+WASO; Figs 2 and 3) from participants 1:1, 1:2, 1:3, 1:4, 2:1 and 2:3 indicated a reduction in undesired time spent awake between baseline (A) and treatment phase (B). The reported undesired time awake was either stable or had improved from post-treatment to the 4-month follow-up. Follow-up data for participants 2:2 and 2:3 were not obtained. The data from participant 2:2 indicated an increase in reported undesired time awake between baseline (A) and treatment phase (B) (see Figs 2 and 3). The same pattern was observed in NAP scores and weekly insomnia measures 

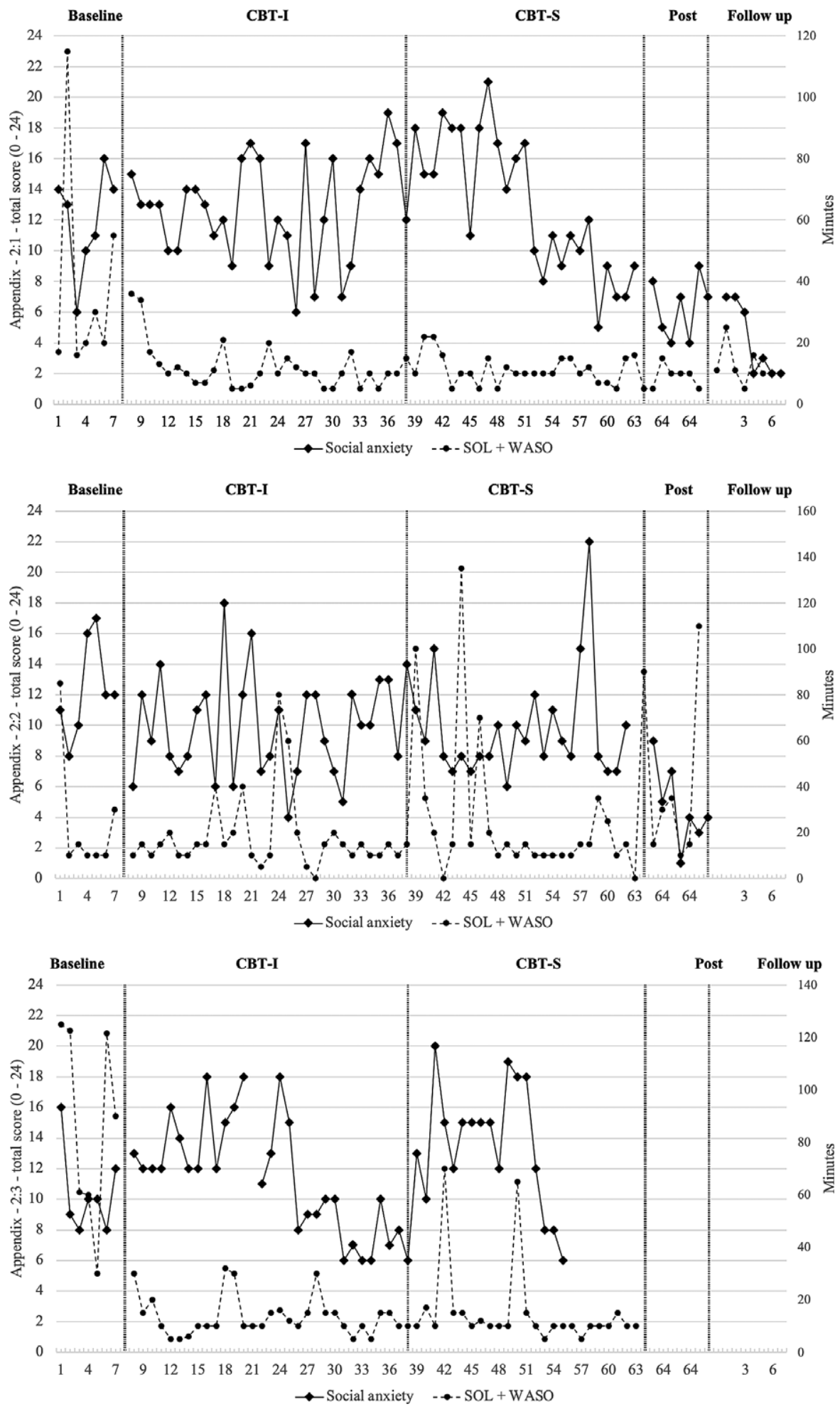

Figure 3. Social anxiety and insomnia symptoms over time for treatment sequence 2 . 
Table 2. Effect sizes (non-overlap of all pairs) of daily measures

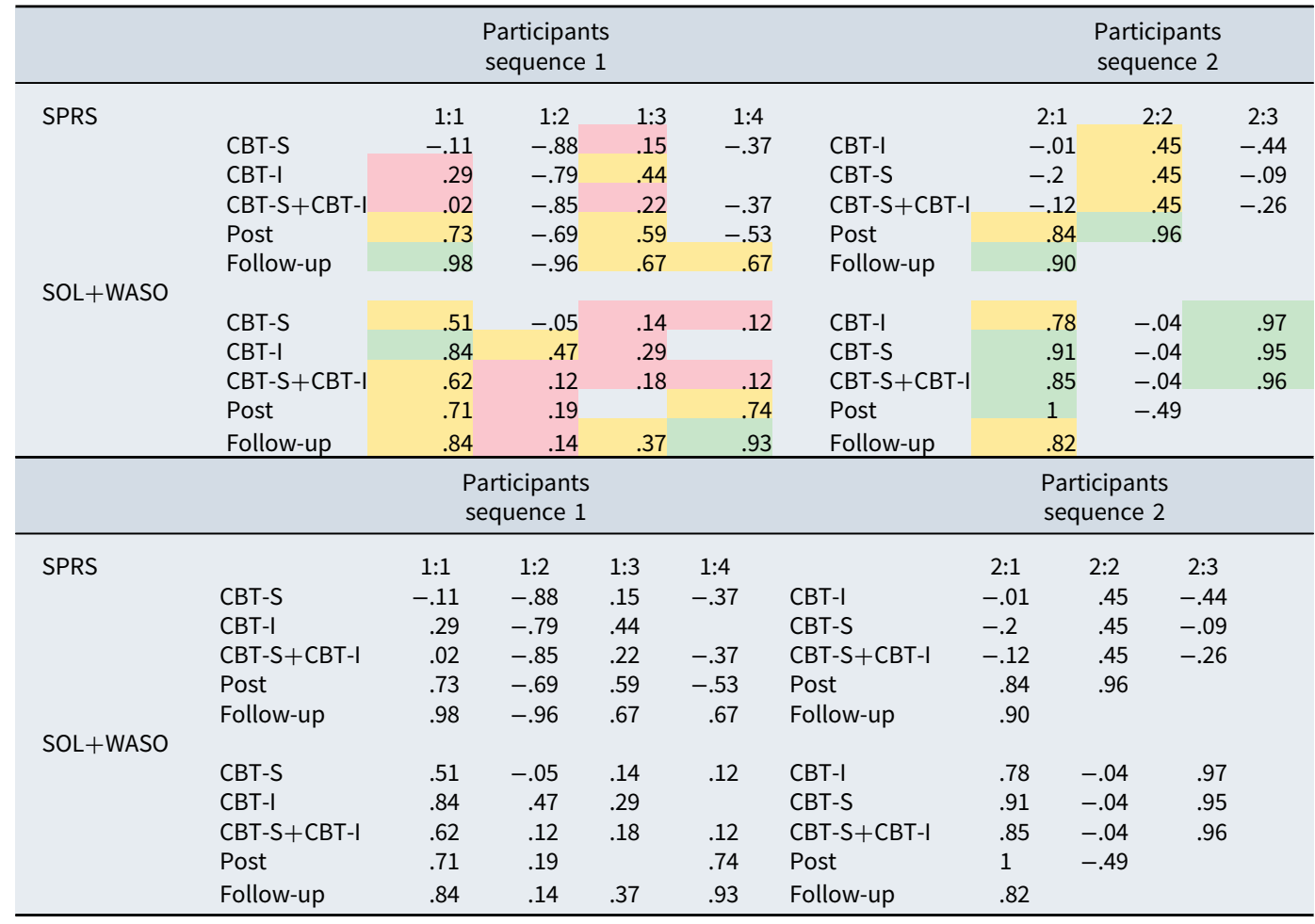

Small effect size

Medium effect size

Large effect size.

Non-overlap of all pairs (NAP) on daily measures of social anxiety (modified short version of the Social Phobia Rating Scale [SPRS]) and undesired time awake (sleep onset latency [SOL]+wake after sleep onset [WASO]) between baseline and following sub-phases, including CBT-S+CBT-I. Negative values indicate deterioration. Measurements are presented in chronological order for each sequence.

with ISI (see Tables 2 and 3). Participant 2:2 showed improvement on ISI (14 to 3) but a deterioration in daily measures at post-treatment. A deviation in the opposite direction was noted for participants 1:3 and 1:4, whose ISI scores deteriorated between post-treatment and follow-up ( 2 to 16 and 11 to 12, respectively; Table 3), whereas the NAP scores of daily measures improved (Table 2). However, post-treatment data from daily measures were missing for participant 1:3. A comparison was therefore made between baseline (A) and the daily measures from the CBT-I treatment).

\section{Does sequential treatment based on CBT-S and CBT-I decrease depressive symptoms?}

Weekly measurements with the MADRS-S showed reduced scores (indicating reduced selfreported depressive symptoms) for participants 1:1, 1:2, 1:3, 2:1 and 2:2 between baseline (A) and post-treatment. Post-treatment data from participant 2:3 was missing. Participants 1:1, 1:2 and 2:1 were either stable or had improved between post-treatment and the 4-month followup. Follow-up data for participants 2:2 and 2:3 were not obtained (see Table 3). The opposite pattern was observed for participants 1:2 and 1:4, with increased MADRS scores between post-treatment and the 4-month follow-up. Neither the post-treatment data nor the follow-up data were obtained for participant 2:3. However, decreased MADRS scores between baseline (A) and the last sequence for participant 2:3 was noted. 
Table 3. Clinical outcome measures

\begin{tabular}{|c|c|c|c|c|c|c|c|c|c|}
\hline & & \multicolumn{3}{|c|}{ Participants sequence 1} & \multirow[b]{2}{*}{$1: 4$} & & \multicolumn{3}{|c|}{ Participants sequence 2} \\
\hline & & $1: 1$ & $1: 2$ & $1: 3$ & & & $2: 1$ & $2: 2$ & $2: 3$ \\
\hline \multicolumn{10}{|l|}{ LSAS-SR } \\
\hline & Baseline & 100 & 57 & 61 & 67 & Baseline & 92 & 71 & 40 \\
\hline & CBT-S & 98 & 62 & 56 & 68 & CBT-I & 94 & 71 & 43 \\
\hline & CBT-I & 69 & 56 & 42 & - & CBT-S & 95 & 76 & 60 \\
\hline & Post & 45 & 48 & 47 & 44 & Post & 74 & 59 & - \\
\hline & Follow-up & 29 & 51 & 28 & 35 & Follow-up & 42 & - & - \\
\hline & Dif & -55 & -9 & -14 & -23 & Dif & -18 & -12 & 20 \\
\hline & Dif \% & $-55 \%$ & $-16 \%$ & $-23 \%$ & $-34 \%$ & Dif \% & $-20 \%$ & $-17 \%$ & $50 \%$ \\
\hline \multicolumn{10}{|l|}{ ISI } \\
\hline & Baseline & 20 & 15 & 16 & 18 & Baseline & 18 & 14 & 21 \\
\hline & CBT-S & 20 & 17 & 14 & 19 & CBT-I & 13 & 10 & 7 \\
\hline & CBT-I & 12 & 12 & 15 & - & CBT-S & 9 & 8 & 4 \\
\hline & Post & 10 & 11 & 2 & 11 & Post & 5 & 3 & - \\
\hline & Follow-up & 6 & 8 & 16 & 21 & Follow-up & 9 & - & - \\
\hline & Dif & -10 & -4 & -14 & -7 & Dif & -13 & -11 & -17 \\
\hline & Dif \% & $-50 \%$ & $-27 \%$ & $-88 \%$ & $-39 \%$ & Dif \% & $-72 \%$ & $-78 \%$ & $-80 \%$ \\
\hline \multicolumn{10}{|l|}{ MADRS-S } \\
\hline & Baseline & 24 & 11 & 12 & 26 & Baseline & 22 & 14 & 15 \\
\hline & CBT-S & 19 & 12 & 15 & 25 & CBT-I & 19 & 13 & 11 \\
\hline & CBT-I & 9 & 11 & 17 & - & CBT-S & 12 & 10 & 12 \\
\hline & Post & 7 & 9 & 8 & 21 & Post & 8 & 5 & - \\
\hline & Follow-up & 7 & 13 & 7 & 29 & Follow-up & 4 & - & - \\
\hline & Dif & -17 & -2 & -4 & -5 & Dif & -14 & -9 & -3 \\
\hline & Dif \% & $-71 \%$ & $-16 \%$ & $-35 \%$ & $-19 \%$ & Dif \% & $-64 \%$ & $-65 \%$ & $-20 \%$ \\
\hline
\end{tabular}

Mean scores of weekly measurements for each phase and instrument, difference (Dif) between baseline mean and post (or last available) measurement in absolute score and percentage. a-c marks severity through clinical categories: The LSAS; $30=$ probably social phobia (a), $>60=$ probably generalized social phobia (b). ISI; 0-7 = absence of insomnia, 8-14 = subthreshold insomnia (a), 15-21= moderate insomnia (b), $22-28=$ severe insomnia (c).

\section{Discussion}

The aim of the current study was to explore the effects of sequential CBT-treatments (CBT-I and CBT-S), primarily on symptoms of social anxiety disorder and insomnia disorder, and secondarily on symptoms of depression. In summary, the majority of participants improved in sleep, social anxiety and depressive symptoms. However, the treatment effects were mixed and participants exhibited varying levels of improvement (and deterioration) for all three disorders. Hence, sequential CBT-treatments are potentially effective in decreasing symptoms of social anxiety and insomnia for people with this understudied co-morbidity, and symptoms of depression do potentially decrease along with the primary symptoms.

The post-treatment measurements indicate that the sequential treatments are more effective for insomnia than for social anxiety, with six of the seven participants showing improvements. However, some reported deviations and fluctuations over time, and there were contradictory data between daily and weekly measures for one participant. One explanation could be that the sleep diary captures time-related insomnia symptoms night by night, whereas the weekly measure with ISI captures symptoms and difficulties related to insomnia more broadly. Taken together, these results are in line with previous studies demonstrating that CBTI has an effect on insomnia disorder with co-morbid psychiatric or medical conditions (Wu et al., 2015) as well as with co-morbid social anxiety disorder (Tang, 2010). The changes in undesired time awake in this study seem to co-occur with the CBT-I phase rather than during the CBT-I phase, which suggests that CBT-I in itself is effective at reducing symptoms of insomnia disorder in people with co-morbid social anxiety disorder.

The results concerning the treatment effects on social anxiety are more difficult to interpret. Fewer participants reported improvements in social anxiety symptoms than in insomnia symptoms. Participants 1:1, 1:3, 2:1 and 2:2 responded well or relatively well to the treatment, as 
demonstrated by both daily and weekly measures of social anxiety. However, the data from the remaining three participants $(1: 2,1: 4$ and 2:3) did not demonstrate any clear improvements except for participant 1:4, who demonstrated improvements in both daily and weekly measures at the 4-month follow-up. From the four participants that displayed improvements, the change seems to be gradual but not within CBT-S. This suggests that the improvement of social anxiety disorder could be attributed to CBT-S with a delayed effect. As the exposure module is found in the latter part of the CBT-S protocol it is not far-fetched to imagine that participants who experienced some initial success would keep approaching previously aversive stimuli in a generalized manner. Another explanation might be that both treatments are needed when treating co-morbid social anxiety disorder and insomnia. It is possible to retrospectively imagine pathways such as a decrease in rumination or worry at night-time, or that one would simply be more wellrested. The results from Tang (2010), however, indicated no decrease in social anxiety from CBT-I in the same population. It is interesting to note that the four participants who improved, together with participant 1:4, reported severe levels of social anxiety before treatment. This suggests that sequential CBT treatments may be more effective for severe social anxiety than for mild or moderate social anxiety. Although comparisons between single-case experimental designs and group designs should be done with great caution, the distribution of the results nevertheless align with those of previous studies on CBT-S whose main components were exposure and cognitive restructuring (Loerinc et al., 2015; Rodebaugh et al., 2004). It is important to note that CBT-S was compressed into six sessions instead of nine in the current study, and that several of the participants (1:1, 1:4, 2:1, 2:2 and 2:3) had additional co-morbid psychiatric diagnoses.

Concerning depression, these results are also inconclusive and with the current design, inferences about treatment effects are therefore difficult to make. All participants who reported data at posttreatment displayed a decrease in MADRS-S scores that ranged between 16 and $71 \%$. However, at the 4-month follow-up, two out of five participants deteriorated (1:2 and 1:4) from the baseline and two participants did not report any follow-up data (2:2 and 2:3). It is interesting to note, however, that the participants with the largest relative decrease in reported depressive symptoms between baseline and post-treatment measurements (1:1, 1:3, 2:1 and 2:2) were the same participants with the highest decrease in social anxiety symptoms between baseline and posttreatment measurements through visual inspection of daily measures from SPRS. This is partly supported by the previous findings proposing depression as a mediator between insomnia disorder and social anxiety disorder (Buckner et al., 2008). As no treatment for depression was administered in the current study, this suggests the possibility of a more complex bidirectional or just coincidental - relationship between these disorders.

In the current study, only three out of 10 participants completed all of the daily measure entries during baseline, treatment, post-treatment, and follow-up. Furthermore, only half of the participants completed the treatment without modifications. However, drop-out rates are similar to those reported previously (Fernandez et al., 2015), although proportions must, once again, be interpreted carefully when comparisons are made between group studies and singlecase experimental studies. The current study was primarily focused on internal validity, but questions of ecological validity in clinical studies with a heavy load of weekly and daily measurements are warranted in future research, especially as the idiographic scientist/ practitioner perspective attracts more interest (Hayes et al., 2019).

Some limitations with the current study must be considered. First, relatively large fluctuations were observed during the baseline (A) and treatment (B) phases, which lower the internal validity and render causal conclusions based on visual analysis more difficult to draw. Furthermore, most fluctuations did not stabilize during baseline, which made it more challenging to assess trends and effect sizes. Statistical analyses of effect sizes with NAP were used to address the uncertainty introduced by these fluctuations. Future studies are encouraged to use sufficiently long baseline periods to stabilize fluctuations and to randomize the duration of baseline periods in order to minimize further threats to internal validity, such as history, which cannot be 
excluded with the current design. However, no participants in this study reported any major events during the baseline or treatment phases.

Second, the daily social anxiety measure consisted of a modified version of SPRS (in which three of the original five items were used), which increases the uncertainty of some of its psychometric properties. SPRS is developed for group studies wherein the purpose is to measure differences between people over more than 7 days, rather than measuring changes within a person over 1 day. However, the selected items are theoretically relevant to people with social anxiety, namely, distress, avoidance and self-focus. However, it remains unknown to what extent these three items correspond to the problems reported by each participant.

Third, the missing data and drop-out rates are problematic in that they render accurate interpretations of the results more difficult. Future studies should aim to systematically collect more qualitative data to better assess treatment feasibility, especially when scarcely investigated populations and treatments, or combinations of treatments, are investigated with small sample sizes. Fourth, no measures of adherence besides attendance were used. Future studies should track participant adherence to assignments and therapist adherence to protocols. However, treatment manuals were used and reading assignments were given to increase internal validity. Fifth, the two treatments (CBT-I and CBT-S) were given one after the other, which means that delayed effects cannot be ruled out, hence diffusion of treatments. However, an attempt to mitigate this was made by randomizing participants to the different sequences. No clear differences in outcome attributed to the order of treatments were detected even though the ideographic design does not really allow such comparisons.

Finally, some strengths with the current study should also be highlighted. First, participants in single-case experiments double as their own control. By the repeated measurements in our design, threats to internal validity, such as testing and statistical regression, could be explored. Second, additional support for internal validity and to some extent also external validity is that the initial case could be said to have been replicated six times. Third, the participants were randomized to each sequence to control for order effects. Fourth, by using both visual analysis and statistical analysis (NAP), we have a relatively high analytical validity. It is especially important to use a statistical analysis method in addition to the visual analysis when the daily measures show a great deal of fluctuations, as in our case. Fifth, the daily measures are supplemented with weekly measures with questionnaires with sufficient psychometric properties as well as norm data, which allow for somewhat more safe conclusions concerning symptoms of insomnia disorder and social anxiety disorder. Sixth, we tried to reduce effects of social desirability by letting the participants submit their completed questionnaires in sealed envelopes. Last, in order to strengthen internal validity, treatment protocols for both treatments were used and the participants were thoroughly screened with MINI.

In conclusion, the sequential treatments with CBT-I and CBT-S seem to decrease symptoms of insomnia and social anxiety in people with co-morbid insomnia disorder and social anxiety disorder. This study takes a first step to explore one passable treatment strategy for co-morbid insomnia and social anxiety. Future research should strive to increase experimental control and to further assess the relationship between insomnia and social anxiety disorder. In relation to the latter, it would be of interest for future studies to investigate treatment strategies that address the processes of change through which social anxiety and insomnia symptoms could be related. A mediational study on standard CBT and acceptance and commitment therapy (ACT) for social anxiety disorder found that an initial decrease in negative cognitions predicts better outcomes in both CBT and ACT, whereas an initial decrease in experiential avoidance predicts better outcomes in ACT (Niles et al., 2014). Another study found that changes in self-focused attention was associated with improvements in social anxiety in both individual cognitive therapy (ICT) and cognitive behavioural group therapy (CBGT), whereas improvements in social anxiety in CBGT were also associated with changes in anticipatory and post-event processing (Hedman et al., 2013). Both ACT and unified protocol (UP) addresses emotional/experiential avoidance and recent 
reviews found that both UP and ACT are effective for insomnia disorder (Cassiello-Robbins et al., 2020; Salari et al., 2020) and promising for social anxiety disorder (Cassiello-Robbins et al., 2020; Norton et al., 2015). These results suggest that emotional/experiential avoidance, negative cognitions and self-focused attention may be interesting candidates to examine further as processes of change in psychological treatments for people with co-morbid social anxiety disorder and insomnia disorder.

Acknowledgements. Preliminary data were presented as part of a Master's thesis in Swedish by David Gryphon and Hanna Eriksson at Örebro University in 2016. The current manuscript includes more data and different analyses.

Author contributions. M.T. and A.N.-C. designed the study. M.T., A.N.-C., D.G. and H.E. contributed to the data collection. M.T., D.G. and H.E. conducted preliminary analyses of the data and D.W., S.A., M.T. and A.N.-C. conducted the final analyses. The first draft of the manuscript was created by D.W. All authors critically reviewed the manuscript and approved the final version.

Financial support. This research received no specific grant from any funding agency, commercial or not-for-profit sectors.

Conflicts of interest. The authors declare no conflicts of interest.

Ethical statements. Approval was obtained from the regional ethics review board in Uppsala, Sweden (Dnr: 2016/302). All participants received written information concerning the study and their participation, and they completed an informed consent form. All data were coded and an age range was presented to protect participant anonymity. The clinical trial was registered retrospectively at: $=$ https://www.anzctr.org.au/Trial/Registration/TrialReview.aspx?ACTRN=12617001165370. (clinical registration number: ACTRN12617001165370).

Data availability statement. The data that support the findings of this study are available on reasonable request from the corresponding author, D.W. The data are not publicly available due to information that could compromise research participant privacy/consent.

Supplementary material. To view supplementary material for this article, please visit: https://doi.org/10.1017/ S1352465821000278

\section{References}

American Psychiatric Association (2013). Diagnostic and Statistical Manual of Mental Disorders. American Psychiatric Publishing. https://doi.org/10.1176/appi.books.9780890425596

Amorim, P., Lecrubier, Y., Weiller, E., Hergueta, T., \& Sheehan, D. (1998). DSM-IH-R Psychotic Disorders: procedural validity of the Mini International Neuropsychiatric Interview (MINI). Concordance and causes for discordance with the CIDI. European Psychiatry, 13, 26-34. https://doi.org/10.1016/S0924-9338(97)86748-X

Babson, K. A., Feldner, M. T., Trainor, C. D., \& Smith, R. C. (2009). An experimental investigation of the effects of acute sleep deprivation on panic-relevant biological challenge responding. Behavior Therapy, 40, 239-250. https://doi.org/10. 1016/j.beth.2008.06.001

Baker, S. L., Heinrichs, N., Kim, H. J., \& Hofmann, S. G. (2002). The Liebowitz social anxiety scale as a self-report instrument: a preliminary psychometric analysis. Behaviour Research and Therapy, 40, 701-715. https://doi.org/10. 1016/S0005-7967(01)00060-2

Bandelow, B., \& Michaelis, S. (2015). Epidemiology of anxiety disorders in the 21st century. Dialogues in Clinical Neuroscience, 17, 327-335. https://www.ncbi.nlm.nih.gov/pubmed/26487813

Bastien, C. H., Vallieres, A., \& Morin, C. M. (2001). Validation of the Insomnia Severity Index as an outcome measure for insomnia research. Sleep Medicine, 2, 297-307. https://doi.org/10.1016/S1389-9457(00)00065-4

Belleville, G., Cousineau, H., Levrier, K., \& St-Pierre-Delorme, M. E. (2011). Meta-analytic review of the impact of cognitive-behavior therapy for insomnia on concomitant anxiety. Clinical Psychology Review, 31, 638-652. https://doi. org/10.1016/j.cpr.2011.02.004

Belleville, G., Cousineau, H., Levrier, K., St-Pierre-Delorme, M. E., \& Marchand, A. (2010). The impact of cognitivebehavior therapy for anxiety disorders on concomitant sleep disturbances: a meta-analysis. Journal of Anxiety Disorders, 24, 379-386. https://doi.org/10.1016/j.janxdis.2010.02.010

Blake, M. J., Trinder, J. A., \& Allen, N. B. (2018). Mechanisms underlying the association between insomnia, anxiety, and depression in adolescence: implications for behavioral sleep interventions. Clinical Psychology Review, 63, 25-40. https:// doi.org/10.1016/j.cpr.2018.05.006 
Buckner, J. D., Bernert, R. A., Cromer, K. R., Joiner, T. E., \& Schmidt, N. B. (2008). Social anxiety and insomnia: the mediating role of depressive symptoms. Depression and Anxiety, 25, 124-130. https://doi.org/10.1002/da.20282

Carney, C. E., Ulmer, C., Edinger, J. D., Krystal, A. D., \& Knauss, F. (2009). Assessing depression symptoms in those with insomnia: an examination of the beck depression inventory second edition (BDI-II). Journal of Psychiatric Research, 43, 576-582. https://doi.org/10.1016/j.jpsychires.2008.09.002

Cassiello-Robbins, C., Southward, M. W., Tirpak, J. W., \& Sauer-Zavala, S. (2020). A systematic review of Unified Protocol applications with adult populations: facilitating widespread dissemination via adaptability. Clinical Psychology Review, 101852. https://doi.org/10.1016/j.cpr.2020.101852

Edinger, J. D., \& Carney, C. E. (2014). Overcoming Insomnia: A Cognitive-Behavioral Therapy Approach, Therapist Guide. Oxford University Press. https://doi.org/10.1093/med:psych/9780195365894.001.0001

Eskildsen, A., Hougaard, E., \& Rosenberg, N. K. (2010). Pre-treatment patient variables as predictors of drop-out and treatment outcome in cognitive behavioural therapy for social phobia: a systematic review. Nordic Journal of Psychiatry, 64, 94-105. https://doi.org/10.3109/08039480903426929

Fantino, B., \& Moore, N. (2009). The self-reported Montgomery-Åsberg depression rating scale is a useful evaluative tool in major depressive disorder. BMC Psychiatry, 9, 26. https://doi.org/10.1186/1471-244x-9-26

Fernandez, E., Salem, D., Swift, J. K., \& Ramtahal, N. (2015). Meta-analysis of dropout from cognitive behavioral therapy: magnitude, timing, and moderators. Journal of Consulting and Clinical Psychology, 83, 1108-1122. https://doi.org/10.1037/ ccp0000044

Fresco, D. M., Coles, M. E., Heimberg, R. G., Liebowitz, M. R., Hami, S., Stein, M. B., \& Goetz, D. (2001). The Liebowitz Social Anxiety Scale: a comparison of the psychometric properties of self-report and clinician-administered formats. Psychological Medicine, 31, 1025-1035. https://doi.org/10.1017/s0033291701004056

Furmark, T., Tillfors, M., Everz, P., Marteinsdottir, I., Gefvert, O., \& Fredrikson, M. (1999). Social phobia in the general population: prevalence and sociodemographic profile. Social Psychiatry and Psychiatric Epidemiology, 34, 416-424. https:// doi.org/10.1007/s001270050163

Hayes, S. C., Hofmann, S. G., Stanton, C. E., Carpenter, J. K., Sanford, B. T., Curtiss, J. E., \& Ciarrochi, J. (2019). The role of the individual in the coming era of process-based therapy. Behaviour Research and Therapy, 117, 40-53. https://doi.org/ 10.1016/j.brat.2018.10.005

Hedman, E., Mörtberg, E., Hesser, H., Clark, D. M., Lekander, M., Andersson, E., \& Ljótsson, B. (2013). Mediators in psychological treatment of social anxiety disorder: Individual cognitive therapy compared to cognitive behavioral group therapy. Behaviour Research and Therapy, 51(, 696-705.

Kazdin, A. E. (2011). Single-Case Research Designs: Methods for Clinical and Applied Settings. Oxford, UK: Oxford University Press.

Kessler, R. C., Berglund, P., Demler, O., Jin, R., Merikangas, K. R., \& Walters, E. E. (2005). Lifetime prevalence and age-ofonset distributions of DSM-IV disorders in the National Comorbidity Survey Replication. Archives of General Psychiatry, 62, 593-602. https://doi.org/10.1001/archpsyc.62.6.593

Krystal, A. D. (2012). Psychiatric disorders and sleep. Neurologic Clinics, 30, 1389-1413. https://doi.org/10.1016/j.ncl.2012.08.018

Kushnir, J., Marom, S., Mazar, M., Sadeh, A., \& Hermesh, H. (2014). The link between social anxiety disorder, treatment outcome, and sleep difficulties among patients receiving cognitive behavioral group therapy. Sleep Medicine, 15, 515-521. https://doi.org/10.1016/j.sleep.2014.01.012

LeBlanc, M., Merette, C., Savard, J., Ivers, H., Baillargeon, L., \& Morin, C. M. (2009). Incidence and risk factors of insomnia in a population-based sample. Sleep, 32, 1027-1037. https://doi.org/10.1093/sleep/32.8.1027

Lecrubier, Y., Sheehan, D. V., Weiller, E., Amorim, P., Bonora, I., Sheehan, K. H., Janavs, J., \& Dunbar, G. C. (1997). The Mini International Neuropsychiatric Interview (MINI). A short diagnostic structured interview: reliability and validity according to the CIDI. European Psychiatry, 12, 224-231. https://doi.org/10.1016/S0924-9338(97)83296-8

Loerinc, A. G., Meuret, A. E., Twohig, M. P., Rosenfield, D., Bluett, E. J., \& Craske, M. G. (2015). Response rates for CBT for anxiety disorders: need for standardized criteria. Clinical Psychology Review, 42, 72-82. https://doi.org/10.1016/j.cpr. 2015.08.004

Mallon, L., Broman, J. E., Akerstedt, T., \& Hetta, J. (2014). Insomnia in Sweden: a population-based survey. Sleep Disorders, 2014, 843126. https://doi.org/10.1155/2014/843126

Manolov, R., Losada, J. L., Chacon-Moscoso, S., \& Sanduvete-Chaves, S. (2016). Analyzing two-phase single-case data with non-overlap and mean difference indices: illustration, software tools, and alternatives. Frontiers in Psychology, 7, 32. https:// doi.org/10.3389/fpsyg.2016.00032

Morin, C. M., Belleville, G., Belanger, L., \& Ivers, H. (2011). The Insomnia Severity Index: psychometric indicators to detect insomnia cases and evaluate treatment response. Sleep, 34, 601-608. https://doi.org/10.1093/sleep/34.5.601

Morphy, H., Dunn, K. M., Lewis, M., Boardman, H. F., \& Croft, P. R. (2007). Epidemiology of insomnia: a longitudinal study in a UK population. Sleep, 30, 274-280. https:/www.ncbi.nlm.nih.gov/pubmed/17425223

Neckelmann, D., Mykletun, A., \& Dahl, A. A. (2007). Chronic insomnia as a risk factor for developing anxiety and depression. Sleep, 30, 873-880. https://doi.org/10.1093/sleep/30.7.873 
Niles, A. N., Burklund, L. J., Arch, J. J., Lieberman, M. D., Saxbe, D., \& Craske, M. G. (2014). Cognitive mediators of treatment for social anxiety disorder: comparing acceptance and commitment therapy and cognitive-behavioral therapy. Behavior Therapy, 45, 664-677. https://doi.org/10.1016/j.beth.2014.04.006

Norell-Clarke, A., Jansson-Frojmark, M., Tillfors, M., Hollandare, F., \& Engstrom, I. (2015). Group cognitive behavioural therapy for insomnia: effects on sleep and depressive symptomatology in a sample with comorbidity. Behaviour Research and Therapy, 74, 80-93. https://doi.org/10.1016/j.brat.2015.09.005

Norton, A. R., Abbott, M. J., Norberg, M. M., \& Hunt, C. (2015). A systematic review of mindfulness and acceptance-based treatments for social anxiety disorder. Journal of Clinical Psychology, 71, 283-301. https://doi.org/10.1002/jclp.22144

Ohayon, M. M. (2002). Epidemiology of insomnia: what we know and what we still need to learn. Sleep Medicine Reviews, 6 , 97-111. https://doi.org/10.1053/smrv.2002.0186

Papadimitriou, G. N., \& Linkowski, P. (2005). Sleep disturbance in anxiety disorders. International Review of Psychiatry, 17, 229-236. https://doi.org/10.1080/09540260500104524

Parker, R. I., \& Vannest, K. (2009). An improved effect size for single-case research: nonoverlap of all pairs. Behavior Therapy, 40, 357-367. https://doi.org/10.1016/j.beth.2008.10.006

Parker, R. I., Vannest, K. J., \& Davis, J. L. (2011). Effect size in single-case research: a review of nine nonoverlap techniques. Behavior Modification, 35, 303-322. https://doi.org/10.1177/0145445511399147

Roca, M., Gili, M., Garcia-Garcia, M., Salva, J., Vives, M., Garcia Campayo, J., \& Comas, A. (2009). Prevalence and comorbidity of common mental disorders in primary care. Journal of Affective Disorders, 119, 52-58. https://doi.org/ 10.1016/j.jad.2009.03.014

Rodebaugh, T. L., Holaway, R. M., \& Heimberg, R. G. (2004). The treatment of social anxiety disorder. Clinical Psychology Review, 24, 883-908. https://doi.org/10.1016/j.cpr.2004.07.007

Salari, N., Khazaie, H., Hosseinian-Far, A., Khaledi-Paveh, B., Ghasemi, H., Mohammadi, M., \& Shohaimi, S. (2020). The effect of acceptance and commitment therapy on insomnia and sleep quality: a systematic review. BMC neurology, 20, 1-18. https://doi.org/10.1186/s12883-020-01883-1

Sheehan, D. V., Lecrubier, Y., Sheehan, K. H., Amorim, P., Janavs, J., Weiller, E., Hergueta, T., Baker, R., \& Dunbar, G. C. (1998). The Mini-International Neuropsychiatric Interview (M.I.N.I.): the development and validation of a structured diagnostic psychiatric interview for DSM-IV and ICD-10. Journal of Clinical Psychiatry, 59 (suppl 20), 22 and 33; quiz 34-57. https://www.ncbi.nlm.nih.gov/pubmed/9881538

Sheehan, D. V., Lecrubier, Y., Sheehan, K. H., Janavs, J., Weiller, E., Keskiner, A., Schinka, J., Knapp, E., Sheehan, M. F., \& Dunbar, G. C. (1997). The validity of the Mini International Neuropsychiatric Interview (MINI) according to the SCID$\mathrm{P}$ and its reliability. European Psychiatry, 12, 232-241. https://doi.org/10.1016/S0924-9338(97)83297-X

Svanborg, P., \& Asberg, M. (1994). A new self-rating scale for depression and anxiety states based on the Comprehensive Psychopathological Rating Scale. Acta Psychiatrica Scandinavica, 89, 21-28. https://doi.org/10.1111/j.1600-0447.1994.tb01480.x

Tang, N. K. (2010). Brief CBT-I for insomnia comorbid with social phobia: a case study. Behavioural Cognitive Psychotherapy, 38, 113-122. https://doi.org/10.1017/S1352465809990488

Tillfors, M., Carlbring, P., Furmark, T., Lewenhaupt, S., Spak, M., Eriksson, A., Westling, B. E., \& Andersson, G. (2008). Treating university students with social phobia and public speaking fears: internet delivered self-help with or without live group exposure sessions. Depression and Anxiety, 25, 708-717. https://doi.org/10.1002/da.20416

Uhde, T. W., Cortese, B. M., \& Vedeniapin, A. (2009). Anxiety and sleep problems: emerging concepts and theoretical treatment implications. Current Psychiatry Reports, 11, 269-276. https://doi.org/10.1007/s11920-009-0039-4

van Straten, A., van der Zweerde, T., Kleiboer, A., Cuijpers, P., Morin, C. M., \& Lancee, J. (2018). Cognitive and behavioral therapies in the treatment of insomnia: a meta-analysis. Sleep Medicine Reviews, 38, 3-16. https://doi.org/10.1016/j.smrv.2017. 02.001

Walker, M. P., \& van der Helm, E. (2009). Overnight therapy? The role of sleep in emotional brain processing. Psychological Bulletin, 135, 731-748. https://doi.org/10.1037/a0016570

Wells, A., \& Leahy, R. L. (1998). Cognitive Therapy of Anxiety Disorders: A Practice Manual and Conceptual Guide. Springer. https://doi.org/10.1891/0889-8391.12.4.350

Wu, J. Q., Appleman, E. R., Salazar, R. D., \& Ong, J. C. (2015). Cognitive behavioral therapy for insomnia comorbid with psychiatric and medical conditions: a meta-analysis. JAMA Internal Medicine, 175, 1461-1472. https://doi.org/10.1001/ jamainternmed.2015.3006

Zalta, A. K., Dowd, S., Rosenfield, D., Smits, J. A., Otto, M. W., Simon, N. M., Meuret, A. E., Marques, L., Hofmann, S. G., \& Pollack, M. H. (2013). Sleep quality predicts treatment outcome in CBT for social anxiety disorder. Depression and Anxiety, 30, 1114-1120. https://doi.org/10.1002/da.22170

Cite this article: Wallsten D, Norell-Clarke A, Alfonsson S, Gryphon D, Eriksson H, and Tillfors M (2021). Treating comorbid insomnia and social anxiety disorder with sequential CBT protocols: a single-case experimental study. Behavioural and Cognitive Psychotherapy 49, 641-657. https://doi.org/10.1017/S1352465821000278 